\title{
ESTUDIO Y VALORACIÓN DE LA CARGA TÉRMICA EN AMBIENTES LABORALES EN LA REGIÓN DEL NEA
}

Msc. R. SPOTORNO, Ing. F. GARCÍA, Msc. J. POCHETTINO, Dr. G. FIGUEREDO (*)

\section{Resumen:}

Los especialistas fundamentados en el Decreto Reglamentario N $351 / 79$ de la Ley 19.587 de Higiene y Seguridad en el Trabajo, establecen el régimen laboral de los obreros de las contratistas que desarrollan actividades en diferentes rubros de las industrias. El problema radica que elaborado el plan de trabajo, las contratistas no cumplen con el mismo debido a la elevada carga térmica a la que están expuestos los operarios. En el presente trabajo se realiza la evaluación de la carga térmica para diferentes casos de actividades que los operarios desarrollan en la región del NEA (Nordeste Argentino).

Palabras clave: carga térmica, estudio económico, región NEA

\section{INTRODUCCIÓN}

Cada año aparecen en la prensa titulares que relatan casos de trabajadores que resultaron afectados en el trabajo por golpes de calor, con desenlace grave o fatal. En algunos casos se trata de "accidentes" en los que la carga térmica fue un factor causal.

Detrás de muchos de estos sucesos está también la precariedad: las víctimas suelen ser trabajadores informales, en empresas que carecen de políticas preventivas y de medidas de protección. En éstas, no suele haber responsables de la prevención de los trabajadores, aún en condiciones de cargas térmicas elevadas, suelen soportar su malestar. La actitud en estos casos suele ser considerar que el calor es un factor "natural" derivado de la tarea o del clima.

En contraste con los ambientes industriales, en los que el riesgo de estar expuesto a carga térmica suele estar bien reconocido (en particular en aquellos en los que se utiliza calor como parte del proceso productivo) los trabajadores que realizan sus tareas al aire

(*) GITEA-Grupo de Investigación en Tecnologías Energéticas Apropiadas. Universidad Tecnológica Nacional.

Facultad Regional Resistencia. French 414.3500. Resistencia-Chaco. República Argentina

Tel: +54 3624 432928/Fax: +54 3624 432683/e-mail: rubenspotorno@yahoo.com 
libre no suelen estar protegidos a no evaluarse la prevención necesaria frente al riesgo. En muchas situaciones en trabajos realizados en el exterior se desatienden los modos tradicionales de evitar o reducir la exposición a la carga térmica (paradas voluntarias, "horarios de verano", paradas durante las horas centrales del día; abrigo del sol con sombras o creación de éstas, uso de botijos, ropa ligera, etc.) y en su lugar se imponen condiciones inaceptables por "imperativos económicos". E1 resultado es que existe un abanico cada vez más amplio de actividades potencialmente afectadas por este riesgo.

La existencia de calor en el ambiente laboral constituye frecuentemente una fuente de problemas que se traducen en quejas por falta de confort, bajo rendimiento en el trabajo y, en ocasiones, riesgos para la salud. El estudio del ambiente térmico requiere el conocimiento de una serie de variables del ambiente, del tipo de trabajo y del individuo.

La carga térmica corresponde a la carga neta de calor a la que los trabajadores están expuestos y que resulta de la contribución combinada de las condiciones ambientales del lugar donde trabajan, la actividad física que realizan y las características de la ropa que llevan. La sobrecarga térmica es la respuesta fisiológica del cuerpo humano al estrés térmico y corresponde al coste que le supone al cuerpo humano el ajuste necesario para mantener la temperatura interna en el rango adecuado. Entre los factores que se miden y que determinan el estrés térmico potencial se incluyen: la temperatura del aire, la humedad relativa, la velocidad del aire, la radiación, la actividad metabólica y el tipo de ropa (emisividad y radiación de la misma). La medición de estos factores permite determinar las demandas térmicas internas y externas que dan lugar a la termorregulación del cuerpo humano. En definitiva, las mediciones de carga térmica constituyen la base de la evaluación del ambiente térmico de trabajo, pero no predicen de manera exacta si las condiciones bajo las que está trabajando una persona no suponen un riesgo para su salud.

En el presente trabajo se exponen diferentes casos analizados sobre la carga térmica a la que están expuestas los trabajadores para distintas condiciones de trabajo en la región del NEA, con las posibles consecuencias a producir en el operario y se analiza los mismos desde el punto de vista del costo económico, respecto al tiempo extra a trabajar para cumplir con el cronograma establecido. Además se plantea el estudio debido al desconocimiento de las contratistas provenientes de la región central y sur del país, del efecto que genera las elevadas temperaturas durante el desarrollo de las diferentes actividades a realizar en la región del NEA.

\section{MATERIALES Y MÉTODOS}

Con la finalidad de realizar el análisis de la carga térmica en diferentes ambientes de trabajo en la región del NEA, se utilizó un medidor marca TES modelo1369 como se observa en la Figura 1. 
Figura 1: Medidor de Estrés Térmico

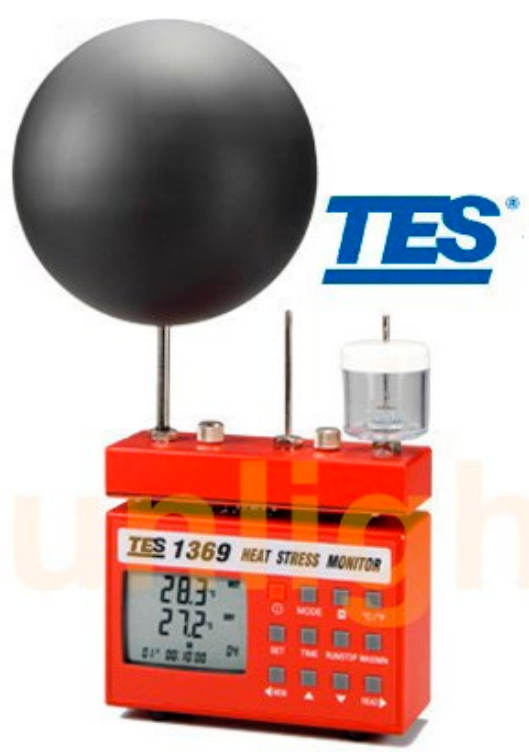

A continuación se presentan distintos casos de mediciones para diferentes condiciones laborales:

\section{Caso 1: Industria del Tanino}

El estudio de estrés térmico se realizó para el $100 \%$ de las actividades bajo cubierta, en la reparación de difusores.

Con el fin de realizar el estudio de estrés térmico, se presenta la metodología y factores a tener en cuenta para su determinación:

\section{Estimación del Calor Metabólico}

Se realiza por medio de tablas según la posición en el trabajo y el grado de actividad.

Se considerará el calor metabólico $(\mathrm{M})$ como la sumatoria del metabolismo basal (MB), y las adiciones derivadas de la posición (MI) y del tipo de trabajo (MII), por lo que:

$\mathbf{M}=\mathbf{M B}+\mathbf{M I}+\mathbf{M I I}$

En donde:

\subsection{Metabolismo basal (MB)}

Se considerará a $\mathrm{MB}=70 \mathrm{~W}$

2.2. Adición derivada de la posición (MI)

\begin{tabular}{|c|c|}
\hline Posición del cuerpo & MI (W) \\
\hline Acostado o sentado & 21 \\
\hline De pie & 42 \\
\hline Caminando & 140 \\
\hline Subiendo pendiente & 210 \\
\hline
\end{tabular}

Tabla 1:Adición derivada de la posición

\subsection{Adición derivada del tipo de trabajo (MII)}

\begin{tabular}{|l|c|}
\hline \multicolumn{1}{|c|}{ Tipo de trabajo } & MII (W) \\
\hline Trabajo manual ligero & 28 \\
\hline Trabajo manual pesado & 63 \\
\hline Trabajo con un brazo: ligero & 70 \\
\hline Trabajo con un brazo: pesado & 126 \\
\hline Trabajo con ambos brazos :ligero & 105 \\
\hline Trabajo con ambos brazos: pesado & 175 \\
\hline Trabajo con el cuerpo: ligero & 210 \\
\hline Trabajo con el cuerpo: moderado & 350 \\
\hline
\end{tabular}

Tabla 2: Adición derivada del tipo de trabajo 
Según CAPITULO 8 - Decreto No 351/79 - ANEXO II. Art.60

\section{LÍMITES PERMISIBLES PARA LA CARGA TÉRMICA}

\section{Valores dados en ${ }^{\circ} \mathrm{C}-\mathrm{TGBH}$}

\begin{tabular}{|l|c|c|c|}
\hline \multicolumn{1}{|c|}{ Régimen de trabajo y descanso } & \multicolumn{3}{|c|}{ Tipo de trabajo } \\
\hline Trabajo continuo & $\begin{array}{c}\text { Liviano } \\
(\text { menos de } 230 \mathrm{~W})\end{array}$ & $\begin{array}{c}\text { Moderado } \\
(230-400 \mathrm{~W})\end{array}$ & $\begin{array}{c}\text { Pesado } \\
(\text { más de 400 W) }\end{array}$ \\
\hline $75 \%$ trabajo y 25 \% descanso, cada hora & 30,0 & 26,7 & 25,0 \\
\hline $50 \%$ trabajo y 50 \% descanso, cada hora & 30,6 & 28,0 & 25,9 \\
\hline $25 \%$ trabajo y 75\% descanso, cada hora & 31,4 & 29,4 & 27,9 \\
\hline
\end{tabular}

Tabla 3: Régimen de trabajo

Donde TGBH: Temperatura de Globo de Bulbo Húmedo

Puesto de Trabajo: Tareas Metalúrgicas en difusores

\begin{tabular}{|c|l|l|}
\hline MB & $70 \mathrm{~W}$ & \multirow{3}{*}{$\mathrm{N}=287 \mathrm{~W}$} \\
\hline MI & $42 \mathrm{~W}$ (Posición del Cuerpo: de pié) & \\
\hline MII & $175 \mathrm{~W}$ (Tipo de Trabajo: con ambos brazos pesado) & \\
\hline
\end{tabular}

Tabla 4: Calor metabólico

\begin{tabular}{|c|c|c|c|}
\hline \multicolumn{4}{|c|}{ MEDICIONES } \\
\hline Hora & TBH $\left({ }^{\circ} \mathrm{C}\right)$ & TG $\left({ }^{\circ} \mathrm{C}\right)$ & TGBH $\left({ }^{\circ} \mathrm{C}\right)$ \\
\hline $10: 30$ & 29,7 & 32,0 & 30,4 \\
\hline $11: 00$ & 29,9 & 32,0 & 30,5 \\
\hline $11: 30$ & 30,3 & 32,2 & 30,9 \\
\hline $12: 00$ & 30,5 & 32,1 & 31,0 \\
\hline $12: 30$ & 30,8 & 32,2 & 31,2 \\
\hline $13: 00$ & 31,0 & 32,5 & 31,5 \\
\hline $13: 30$ & 31,2 & 32,6 & 31,6 \\
\hline $14: 00$ & 31,5 & 33,2 & 32,0 \\
\hline $14: 30$ & 31,6 & 33,2 & 32,1 \\
\hline $15: 00$ & 31,8 & 33,0 & 32,2 \\
\hline $15: 30$ & 32,0 & 33,0 & 32,3 \\
\hline $16: 00$ & 32,0 & 33,5 & 32,5 \\
\hline $16: 30$ & 32,3 & 34,0 & 32,8 \\
\hline $17: 00$ & 32,4 & 34,1 & 32,9 \\
\hline
\end{tabular}

La Tabla 5 presenta las mediciones realizadas con el medidor de estrés térmico, durante el desarrollo de las actividades realizadas por los operarios en la industria de tanino.

Tabla 5: Mediciones

\begin{tabular}{|l|c|}
\hline \multirow{2}{*}{\multicolumn{1}{|c|}{ Régimen de trabajo y descanso }} & Tipo de trabajo \\
\cline { 2 - 2 } & Moderado(230-400 W) \\
\hline Trabajo continuo & 26,7 \\
\hline $75 \%$ trabajo y $25 \%$ descanso, cada hora & 28,0 \\
\hline $50 \%$ trabajo y $50 \%$ descanso, cada hora & 29,4 \\
\hline $25 \%$ trabajo y $75 \%$ descanso, cada hora & 31,1 \\
\hline
\end{tabular}

Calor Metabólico = $287 \mathrm{~W}$

Tabla 6: Régimen de trabajo 
Para el día que se realizó la evaluación de la carga térmica, se llegan a las siguientes conclusiones que se observan en la gráfica de la figura 2.

- Desde el horario de ingreso hasta las 12,00 hs: $50 \%$ trabajo y $50 \%$ descanso, cada hora. - Desde las 12,30 hs hasta la finalización de la jornada laboral: 25 \% trabajo y 75 \% descanso, cada hora.

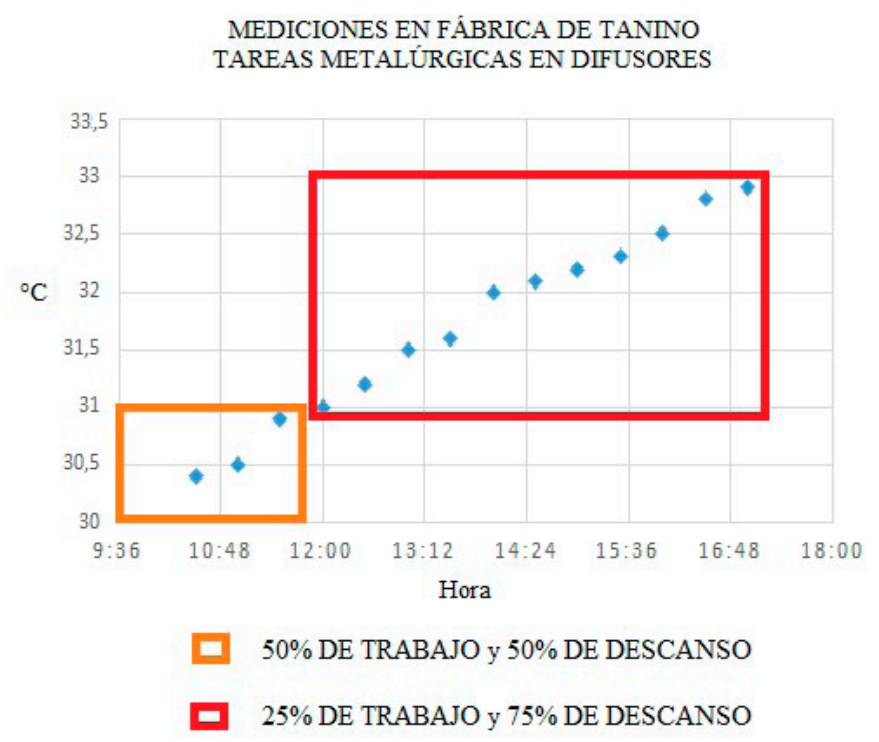

Figura 2: Mediciones de TGBH

Caso 2: Industria de Producción de Harina de Huesos

En este caso también el estudio de estrés térmico se realizó para el $100 \%$ de las actividades bajo cubierta.

Puesto de Trabajo: Tareas de Mantenimiento Generales

\begin{tabular}{|c|l|l|}
\hline MB & $70 \mathrm{~W}$ & \multirow{3}{*}{$\mathrm{M}=217 \mathrm{~W}$} \\
\hline MI & $42 \mathrm{~W}$ (Posición del Cuerpo: de pié) & \\
\hline MII & $105 \mathrm{~W}$ (Tipo de Trabajo: con ambos brazos ligero) & \\
\hline
\end{tabular}

Tabla 7: Calor metabólico

\begin{tabular}{|c|c|c|c|}
\hline \multicolumn{4}{|c|}{ MEDICIONES } \\
\hline Hora & TBH $\left({ }^{\circ} \mathrm{C}\right)$ & TG $\left({ }^{\circ} \mathrm{C}\right)$ & TGBH $\left({ }^{\circ} \mathrm{C}\right)$ \\
\hline $09: 30$ & 28,9 & 31,0 & 30,0 \\
\hline $10: 00$ & 29,6 & 32,0 & 30,3 \\
\hline $10: 30$ & 29,9 & 31,9 & 30,4 \\
\hline $11: 00$ & 30,3 & 32,2 & 30,9 \\
\hline
\end{tabular}




\begin{tabular}{|l|l|l|l|}
\hline $11: 30$ & 30,3 & 32,4 & 31,1 \\
\hline $12: 00$ & 30,6 & 32,7 & 31,3 \\
\hline $12: 30$ & 31,0 & 32,5 & 31,5 \\
\hline $13: 00$ & 31,0 & 32,5 & 31,5 \\
\hline $13: 30$ & 31,3 & 32,6 & 31,7 \\
\hline $14: 00$ & 31,5 & 33,4 & 32,1 \\
\hline $14: 30$ & 31,7 & 33,5 & 32,2 \\
\hline $15: 00$ & 32,0 & 33,0 & 32,3 \\
\hline $15: 30$ & 32,0 & 33,0 & 32,3 \\
\hline $16: 00$ & 32,0 & 33,7 & 32,7 \\
\hline $16: 30$ & 32,5 & 34,1 & 32,9 \\
\hline $17: 00$ & 32,5 & 34,1 & 32,9 \\
\hline
\end{tabular}

Tabla 8: Mediciones

En la tabla 8 se observa las mediciones durante la jornada laboral en la planta de producción de harina de huesos.

Calor Metabólico $=217 \mathrm{~W}$

\begin{tabular}{|l|c|}
\hline \multirow{2}{*}{ Régimen de trabajo y descanso } & Tipo de trabajo \\
\cline { 2 - 2 } & Liviano ( menos de 230 W) \\
\hline Trabajo continuo & 30,0 \\
\hline $75 \%$ trabajo y $25 \%$ descanso, cada hora & 30,6 \\
\hline $50 \%$ trabajo y $50 \%$ descanso, cada hora & 31,4 \\
\hline $25 \%$ trabajo y $75 \%$ descanso, cada hora & 32,2 \\
\hline
\end{tabular}

Tabla 9: Régimen de trabajo

Para el día seleccionado para realizar la evaluación de la carga térmica, y el puesto de trabajo estudiado, se llegan a las siguientes conclusiones:

- Desde el horario de ingreso hasta las 11,00 hs: Trabajo Continuo.

- Desde las 11,00 hs hasta las 12,30 hs: $75 \%$ trabajo y $25 \%$ descanso, cada hora.

- Desde las 12,30 hs hasta las 14,00 hs: $50 \%$ trabajo y $50 \%$ descanso, cada hora.

- Desde las 15,00 hs hasta la finalización de la jornada laboral: $25 \%$ trabajo y $75 \%$ descanso, cada hora. 


\section{Caso 3: Planta de Almacenamiento de Combustibles}

Las actividades que desarrollaron los operarios durante el estudio de estrés térmico, se realizaba $50 \%$ bajo cubierta y $50 \%$ al aire libre.

Puesto de Trabajo: Tareas de Mantenimiento Generales

\begin{tabular}{|c|l|l|}
\hline MB & $70 \mathrm{~W}$ & \multirow{3}{*}{ M=287 W } \\
\hline MI & $42 \mathrm{~W}$ (Posición del Cuerpo: de pie) & \\
\hline MII & $175 \mathrm{~W}$ (Tipo de Trabajo: con ambos brazos pesado) & \\
\hline
\end{tabular}

Tabla 10: Calor metabólico

\begin{tabular}{|c|c|c|c|}
\hline \multicolumn{4}{|c|}{ MEDICIONES } \\
\hline Hora & TBH $\left({ }^{\circ} \mathrm{C}\right)$ & TG $\left({ }^{\circ} \mathrm{C}\right)$ & TGBH $\left({ }^{\circ} \mathrm{C}\right)$ \\
\hline $09: 00$ & 29,7 & 32,0 & 30,4 \\
\hline $09: 30$ & 29,9 & 32,0 & 30,5 \\
\hline $10: 00$ & 30,3 & 32,2 & 30,9 \\
\hline $10: 30$ & 30.2 & 34.7 & 31.2 \\
\hline $11: 00$ & 30.2 & 34.7 & 31.2 \\
\hline $11: 30$ & 31,0 & 32,5 & 31,3 \\
\hline $12: 00$ & 31,0 & 32,5 & 31,5 \\
\hline $12: 30$ & 31,2 & 32,6 & 31,6 \\
\hline $13: 00$ & 31,2 & 32,6 & 31,6 \\
\hline $13: 30$ & 31.3 & 33.1 & 31.8 \\
\hline $14: 00$ & 31,5 & 33,2 & 32,0 \\
\hline $14: 30$ & 31,6 & 33,2 & 32,1 \\
\hline $15: 00$ & 31,8 & 33,0 & 32,2 \\
\hline $15: 30$ & 31,8 & 33,0 & 32,2 \\
\hline $16: 00$ & 32,3 & 34,0 & 32,8 \\
\hline $16: 30$ & 32,4 & 34,1 & 32,9 \\
\hline $17: 00$ & 32,5 & 34,0 & 33,0 \\
\hline
\end{tabular}

Tabla 11: Mediciones

En la Tabla 11 se observa el registro de las mediciones realizadas durante el desarrollo de las actividades en la planta de almacenamiento de combustibles.

Calor Metabólico $=287 \mathrm{~W}$

\begin{tabular}{|l|c|}
\hline \multirow{2}{*}{ Régimen de trabajo y descanso } & Tipo de trabajo \\
\cline { 2 - 2 } & Moderado(230-400 W) \\
\hline Trabajo continuo & 26,7 \\
\hline $75 \%$ trabajo y 25\% descanso, cada hora & 28,0 \\
\hline $50 \%$ trabajo y 50 \% descanso, cada hora & 29,4 \\
\hline $25 \%$ trabajo y 75\% descanso, cada hora & 31,1 \\
\hline
\end{tabular}

Tabla 12: Régimen de trabajo 
Para el día seleccionado para realizar la evaluación de la carga térmica, y el puesto de trabajo estudiado, se obtiene las siguientes conclusiones:

- Desde el horario de ingreso hasta las 10,30 hs: 50 \% trabajo y $50 \%$ descanso, cada hora.

- Desde las 10,30 hs hasta la finalización de la jornada laboral: 25 \% trabajo y $75 \%$ descanso, cada hora.

\section{Caso 4: Proceso de Pintado de Superficies Interiores y Exteriores de Edificios}

Las tareas desarrolladas por los operarios durante el proceso de pintado fueron $50 \%$ bajo cubierta y 50\% al aire libre.

Puesto de Trabajo: Tareas de Pintura Exterior e Interior

\begin{tabular}{|c|l|l|}
\hline MB & $70 \mathrm{~W}$ & \multirow{3}{*}{ M=217 W } \\
\hline MI & $42 \mathrm{~W}$ (Posición del Cuerpo: de pié) & \\
\hline MII & $105 \mathrm{~W}$ (Tipo de Trabajo: con ambos brazos ligero) & \\
\hline
\end{tabular}

Tabla 13: Calor metabólico

\begin{tabular}{|c|c|c|c|}
\hline \multicolumn{4}{|c|}{ MEDICIONES } \\
\hline Hora & TBH $\left({ }^{\circ} \mathrm{C}\right)$ & TG $\left({ }^{\circ} \mathrm{C}\right)$ & TGBH $\left({ }^{\circ} \mathrm{C}\right)$ \\
\hline $10: 00$ & 30.2 & 34.7 & 31.2 \\
\hline $10: 30$ & 31,0 & 32,5 & 31,3 \\
\hline $11: 00$ & 31,2 & 32,6 & 31,4 \\
\hline $11: 30$ & 31,5 & 33,2 & 32,0 \\
\hline $12: 00$ & 30,6 & 32,7 & 31,3 \\
\hline $12: 30$ & 31,0 & 32,5 & 31,5 \\
\hline $13: 00$ & 31,0 & 32,5 & 31,5 \\
\hline $13: 30$ & 31,3 & 32,6 & 31,7 \\
\hline $14: 00$ & 31,5 & 33,2 & 32,0 \\
\hline $14: 30$ & 31,6 & 33,2 & 32,1 \\
\hline $15: 00$ & 31,8 & 33,0 & 32,2 \\
\hline $15: 30$ & 32,3 & 34,0 & 32,8 \\
\hline $16: 00$ & 31,9 & 33,6 & 32,3 \\
\hline $16: 30$ & 31,8 & 33,0 & 32,2 \\
\hline $17: 00$ & 31,5 & 33,2 & 32,0 \\
\hline
\end{tabular}

Tabla 14: Mediciones

La Tabla 14 muestra el registro de las mediciones realizadas con el medidor de estrés térmico durante la jornada de pintado.

Calor Metabólico = $217 \mathrm{~W}$ 


\begin{tabular}{|l|c|}
\hline \multirow{2}{*}{ Régimen de trabajo y descanso } & Tipo de trabajo \\
\cline { 2 - 2 } & Liviano ( menos de $230 \mathrm{~W}$ ) \\
\hline Trabajo continuo & 30,0 \\
\hline $75 \%$ trabajo y $25 \%$ descanso, cada hora & 30,6 \\
\hline $50 \%$ trabajo y $50 \%$ descanso, cada hora & 31,4 \\
\hline $25 \%$ trabajo y $75 \%$ descanso, cada hora & 32,2 \\
\hline
\end{tabular}

Tabla 15: Régimen de trabajo

Para el día seleccionado para realizar la evaluación de la carga térmica, y el puesto de trabajo estudiado, se obtienen las siguientes conclusiones:

- Desde el ingreso hasta las 11,00 hs: $75 \%$ trabajo y $25 \%$ descanso, cada hora.

- Desde las 11,00 hs hasta las 15,00 hs: 50 \% trabajo y $50 \%$ descanso, cada hora.

- Desde las 15,00 hs hasta la finalización de la jornada laboral: $25 \%$ trabajo y $75 \%$ descanso, cada hora.

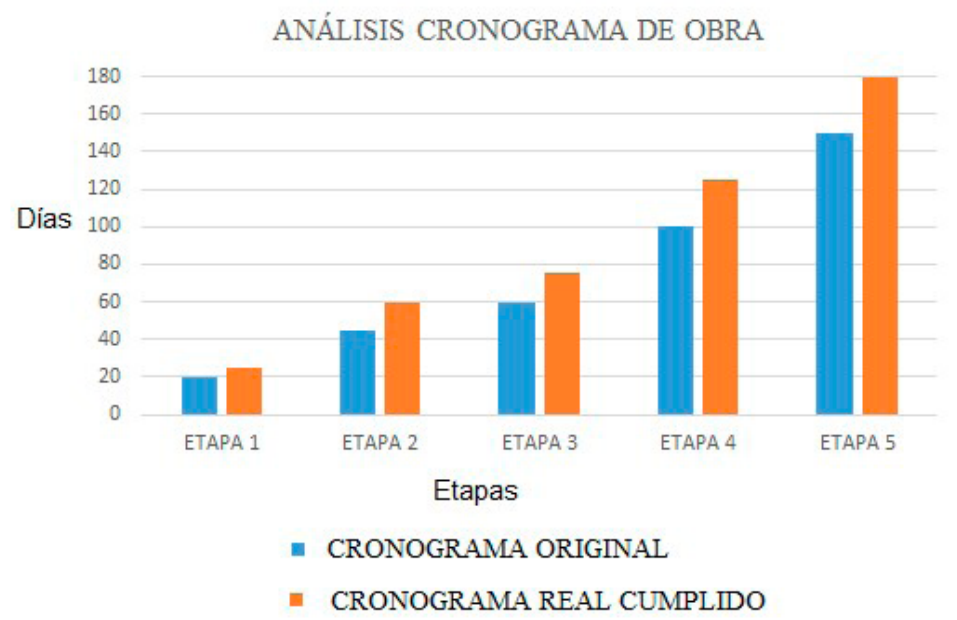

Figura 3: Comparación de cronogramas

En la Figura 3 se observa la diferencia de tiempo transcurrido entre la planificación en el cronograma original (150 días) y el real cumplido de obra (180 días), lo que representa un $20 \%$ en exceso, esto es debido a no tener en cuenta el tiempo elevado de descanso en la jornada laboral y su consecuencia de tener que trabajar días extras para cumplimentar con el cronograma pautado.

Con el fin de realizar un análisis económico teniendo en cuenta los sueldos de los operarios que realizan las mismas actividades, en la zona Centro y NEA del país, se presenta a continuación la gráfica de la Figura 4 . 


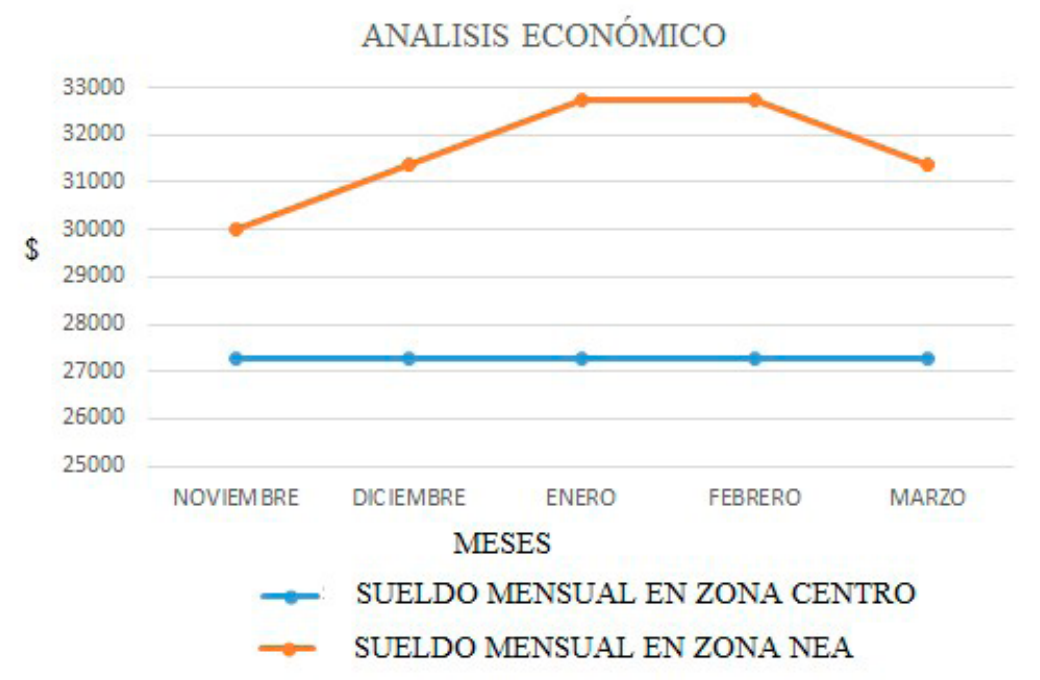

Figura 4: Análisis económico de sueldos

En la Figura 4 se aprecia que el sueldo neto del operario que realiza actividades en la zona centro del país permanece invariable respecto a los meses que se analiza en la gráfica. En cuanto al sueldo del operario que se desempeña en el NEA se observa una diferencia respecto a los meses que se analiza (10\% en noviembre, $15 \%$ en diciembre, $20 \%$ en enero, $20 \%$ en febrero y $15 \%$ en marzo), todos en exceso debido al elevado tiempo de descanso, lo cual produce un aumento del costo de la mano de obra final.

\section{CONCLUSIONES}

De los diferentes casos donde se evaluaron la carga térmica en ambientes de trabajo en el NEA, se lograron obtener las siguientes conclusiones:

Al cumplimentar lo establecido en el decreto 351/79, capítulo 8 Anexo II "carga térmica" se verifica que las tareas se realizan sin perjuicios en la salud de los trabajadores respecto al capítulo analizado.

Aumento del costo al desarrollar una actividad en las provincias de la zona norte del país, comparada con la misma actividad a realizar en las provincias del centro del país.

Inconvenientes generados entre la empresa contratista, proveniente de alguna provincia del centro del país y el comitente, al no poder cumplimentar el cronograma de tareas en tiempo y forma, debido a la no consideración de los tiempos de descanso en la jornada laboral.

Mediante encuestas realizadas a jefes de obras de diferentes contratistas que realizan actividades en la zona del NEA, se determinó que se produce un aumento considerado de inasistencias de los trabajadores cuando las empresas no cumplen con el régimen de trabajo/descanso establecido en el decreto 351/79 capítulo 8 Anexo II "carga térmica". 


\section{ABSTRACT}

The specialists based on the Regulatory Decree No. 351/79 of the Law 19,587 of Health and Safety at Work establish the labor regime of the workers of the contractors who carry out activities in different sectors of the industries. The problem is that when the work plan is drawn up, the contractors do not comply with it because of the high thermal load to which the workers are exposed. In the present work the evaluation of the thermal load is carried out for different cases of activities that the operators develop in the NEA region (Northeast of Argentina).

\section{BIBLIOGRAFÍA}

American Conference of Governmental Industrial Hygienists [1991]. TLVs. threshold limit values and biological exposure indices for 198586, Cincinnati OH: ACGIH pp. 91-98.

Decreto reglamentario 351/79 ANEXO II, Capítulo 8 artículo 60, de la Ley No 19587 Higiene y Seguridad en el Trabajo.

Decreto Presidencial N ${ }^{\circ}$ 11/96. Reglamento para la Industria de la Construcción.

FISO, Fundación Iberoamericana de Seguridad y Salud Ocupacional.www.fiso-web.org
IAS, Instituto Argentino de Seguridad. www.ias.org.

NIOSH [1976]. Standards for occupational exposures to hot environments--proceedings of symposium. Cincinnati, OH: U.S. Department of Health, Education and Welfare, Public Health Service, Center for Disease Control, National Institute for Occupational Safety and Health, HEW(NIOSH) Publication No. 76-100.

Resolución 295/2003 Ministerio de Trabajo, Empleo y Seguridad Social HIGIENE Y SEGURIDAD EN EL TRABAJO. 